\title{
Effect of the Xpert MTB/RIF on the detection of pulmonary tuberculosis cases and rifampicin resistance in Shanghai, China
}

Zheyuan Wu' ${ }^{1}$ Zulma Vanessa Rueda ${ }^{2}$, Tao Li $i^{3}$, Zurong Zhang ${ }^{1}$, Yuan Jiang ${ }^{4}$, Wei Sha ${ }^{5}$, Fangyou Yu ${ }^{6}$, Jing Chen ${ }^{1}$, Qichao Pan ${ }^{7}$, Xin Shen ${ }^{1}$ and Zheng'an Yuan ${ }^{7^{*}}$

\begin{abstract}
Background: Xpert MTB/RIF (Xpert) is an automated molecular test recommended by World Health Organization (WHO) for diagnosis of tuberculosis (TB). This study evaluated the effect of Xpert implementation on the detection of pulmonary TB (PTB) and rifampicin-resistant TB (RR-TB) cases in Shanghai, China.

Methods: Xpert was routinely implemented in 2018 for all presumptive PTB patients. All PTB patients above 15 years-old identified within the Provincial TB Control Program during the first half of each of 2017 and 2018, were enrolled to compare the difference in proportions of bacteriological confirmation, patients with drug susceptibility test (DST) results for rifampicin (ie, DST coverage) and RR-TB detection before and after Xpert's implementation.

Results: A total of 6047 PTB patients were included in the analysis with 1691 tested by Xpert in 2018. Percentages of bacteriological confirmation, DST coverage and RR-TB detection in 2017 and 2018 were $50 \%$ vs. 59\%, 36\% vs. $49 \%$ and $2 \%$ vs. $3 \%$, respectively (all $p$-values $<0.05$ ). Among 1103 PTB patients who completed sputum smear, culture and Xpert testing in 2018, Xpert detected an additional 121 (11\%) PTB patients who were negative by smear and culture, but missed 248 (23\%) smear and/or culture positive patients. Besides, it accounted for an increase of $9 \%$ in DST coverage and 1\% in RR-TB detection. The median time from first visit to a TB hospital to RRTB detection was 62 days (interquartile range -IQR 48-84.2) in 2017 vs. 9 days (IQR 2-45.7) in 2018 (p-value <0.001). In the multivariate model, using Xpert was associated with decreased time to RR-TB detection (adjusted hazard ratio $=4.62,95 \%$ confidence interval: 3.18-6.71).

Conclusions: Integrating Xpert with smear, culture and culture-based DST in a routine setting significantly increased bacteriological confirmation, DST coverage and RR-TB detection with a dramatic reduction in the time to RR-TB diagnosis in Shanghai, China. Our findings can be useful for other regions that attempt to integrate Xpert into routine PTB and RR-TB case-finding cascade. Further study should focus on the identification and elimination of operational level challenges to fully utilize the benefit of rapid diagnosis by Xpert.
\end{abstract}

Keywords: SORT IT, Xpert MTB/RIF, TB, Delay, Rifampicin resistant, Tuberculosis

\footnotetext{
* Correspondence: yuanzhengan@scdc.sh.cn

${ }^{7}$ Shanghai Municiple Center for Disease Control and Prevention, Shanghai,

China

Full list of author information is available at the end of the article
}

(C) The Author(s). 2020 Open Access This article is distributed under the terms of the Creative Commons Attribution 4.0 International License (http://creativecommons.org/licenses/by/4.0/), which permits unrestricted use, distribution, and reproduction in any medium, provided you give appropriate credit to the original author(s) and the source, provide a link to the Creative Commons license, and indicate if changes were made. The Creative Commons Public Domain Dedication waiver (http://creativecommons.org/publicdomain/zero/1.0/) applies to the data made available in this article, unless otherwise stated. 


\section{Background}

China has the second highest tuberculosis (TB) and multidrug-resistant/rifampicin-resistant TB (MDR/RRTB) incidence in the world [1]. Early diagnosis and effective treatment are crucial to stop transmission and to succeed with the End TB strategy [2]. Conventionally, sputum smear microscopy, culture and culture-based drug susceptibility test (DST) are used to diagnose TB and MDR/RR-TB. However, smear microscopy has low sensitivity and specificity compared to culture, culture usually takes weeks to report results, and DST results can take even longer [3].

Xpert $^{\ominus}$ MTB/RIF assay (Cepheid, USA) (Xpert) is a rapid test for $\mathrm{TB}$ diagnosis currently endorsed by the WHO [4], which simultaneously tests for Mycobacterium tuberculosis (MTB) and resistance to rifampicin (RIF), providing results within $2 \mathrm{~h}$. Clinical validation trials have demonstrated high sensitivity and specificity of the test for both MTB and RIF-resistance detection in different settings [4-7]. Several studies have even suggested that Xpert outperforms culture in salivary sputum $[8,9]$ and can detect low-levels of RIF-resistance, which might be missed by culture-based DST [10, 11]. The WHO has recommended that Xpert be the initial test in adults and children with presumptive MDR-TB or HIVassociated TB [4]. The use of Xpert in decentralized settings has significantly increased proportions of bacteriologically confirmed TB and RR-TB and reduced the time to treatment initiation [12-15].

In Shanghai, China, sputum specimens are collected for all presumptive PTB patients to perform smear microscopy and culture. Culture-based drug susceptibility test (DST) for isoniazid $(\mathrm{H})$, rifampicin $(\mathrm{R})$, ethambutol $(\mathrm{E})$, and streptomycin $(\mathrm{S})$ is routinely conducted for culture positive MTB strains to identify MDR-TB [16, 17]. Without any use of the WHO recommended rapid diagnostic methods, the percentage of bacteriologically confirmed PTB patients and DST coverage has been reported at approximately $40 \%$ [18]. Additionally, the median time required to complete culture-based DST is 41 days, which accounts for $23 \%$ of time from TB diagnosis to MDR-TB treatment [16]. Lastly, delayed case identification and the initiation of appropriate treatment may lead to a higher risk of recent transmission and poor TB control [19].

In 2018, Shanghai launched a large scale project funded by the local government to test all presumptive PTB patients using Xpert at their first visit to designated TB hospitals. It aimed to increase bacteriological confirmation and DST coverage among TB patients and to shorten the delay of RR-TB detection. We conducted this study to evaluate the effect of Xpert implementation on the detection of PTB cases and RIF-resistance, including time to RR-TB detection and risk factors associated with RR-TB delay.

\section{Methods}

\section{Study design}

Primarily, this was a cross-sectional study utilizing routinely collected programmatic data for secondary analysis. In addition, we performed a retrospective cohort (time to event analysis) to identify time to RR-TB detection and the factors associated with it.

\section{General setting study population}

There are 30 designated TB hospitals in Shanghai (four referral hospitals and 26 district hospitals) in charge of TB diagnosis and treatment, and more than 240 community health centers in charge of patient management (ie. DOTS). The Xpert project (detailed below) was initiated on January 1st, 2018. By November 2018 when the analysis was performed, only data of the first half of 2018 was available. Therefore, all PTB patients 15 years of age and above identified within the Provincial TB Control Program in the first half of 2018 were included in this study. Patients identified in the first half of 2017 were also enrolled for comparison.

\section{The provincial TB control program}

All presumptive PTB patients (ie, cough for more than 2 weeks) detected in a general hospital or a community health center are referred to the district TB hospital where sputum smear, liquid culture, chest radiograph and other necessary tests are carried out for diagnosis. Culture positive strains are transferred to the TB reference laboratory at Shanghai Center for Disease Control and Prevention (CDC) to conduct a Lowenstein-Jensen culture for species identification and DST for first-line drugs (H/R/E/S); while one referral TB hospital, Shanghai Pulmonary Hospital, conducts liquid culture for species identification and DST for first-line $(\mathrm{H} / \mathrm{R} / \mathrm{E} / \mathrm{S})$ and 3 sec-line drugs (Aminoglycoside/Ofloxacin/Cycloserine). The TB reference laboratory at Shanghai CDC is responsible for the quality control of all TB laboratories in the 30 surrounding TB hospitals.

According to the national standard of diagnosis for PTB [20], confirmed PTB cases include individuals who are sputum smear microscopy positive, culture positive, molecular test positive or individuals who have pulmonary lesions of tuberculosis that have been confirmed by pathological examination (lung biopsy). Those who fail to meet criteria for confirmed PTB are clinically diagnosed if other pulmonary diseases are excluded or their chest radiograph supports active PTB and they have any of the below: 1. PTB signs like cough, expectoration, hemoptysis; 2. Immunology evidence like strong purified protein derivative (PPD) skin test reaction, positive Interferon-gamma release assay (IGRA), positive MTB anti-body test; or 3. extra-pulmonary TB confirmed by pathological examination. 
RR-TB patients are subject to an expert panel [21] for final diagnosis and regimen design. All patients are treated under the supervision of doctors from the local community health center.

\section{The Xpert project}

A total of 17 TB hospitals were equipped with Xpert platforms. Laboratory staff were trained by the reference laboratory of Shanghai CDC on the operation and reporting. Since January 1st, 2018, an Xpert test has been performed for all presumptive PTB patients along with sputum smear and culture at their first visit to one of these TB hospitals. Patients in hospitals without Xpert platforms were transferred to a nearby hospital where Xpert testing was available.
The Global Laboratory Initiative (GLI) and the WHO [3, 22] suggested that patients other than those at high risk of RR-TB (ie, previously treated patients; non-converters [smear positive at end of intensive phase]; and MDR-TB contacts) require a second Xpert test if the initial test was positive for RIF-resistance to control for potential errors. Therefore, new PTB patients in this study were at low risk of RR-TB and those with a RIF-resistant result were offered a second Xpert test to reduce false positive cases.

\section{Data collection and analysis}

Data was extracted from the National TB management information system into Microsoft Excel $^{\circ}$ (Redmond, Washington, USA). A bacteriologically confirmed case was defined as a PTB case with positive results of smear, culture or Xpert. A DST covered case was defined as a

Table 1 Epidemiological characteristics of pulmonary tuberculosis patients notified in Shanghai, China, in the first half of each of 2017 and 2018*

\begin{tabular}{|c|c|c|c|c|}
\hline Characteristics & $\begin{array}{l}\text { Total } \\
\mathrm{n}(\%) \\
(n=6047) \\
\end{array}$ & $\begin{array}{l}\text { PTB patients of } 2017 \\
\mathrm{n}(\%) \\
(n=2938)\end{array}$ & $\begin{array}{l}\text { PTB patients of } 2018 \\
\mathrm{n}(\%) \\
(n=3109)\end{array}$ & $p$-value \\
\hline Sex & & & & 0.912 \\
\hline Female & $1910(31.6)$ & $930(31.7)$ & $980(31.5)$ & \\
\hline Male & $4137(68.4)$ & $2008(68.3)$ & $2129(68.5)$ & \\
\hline Age & & & & 0.198 \\
\hline $15-29$ & $1887(31.2)$ & $904(30.8)$ & $983(31.6)$ & \\
\hline $30-44$ & $1190(19.7)$ & $578(19.7)$ & $612(19.7)$ & \\
\hline $45-59$ & $1171(19.4)$ & $601(20.5)$ & $570(18.3)$ & \\
\hline$>=60$ & $1799(29.8)$ & $855(29.1)$ & $944(30.4)$ & \\
\hline Hospital & & & & 0.928 \\
\hline County-level & $1901(31.4)$ & $922(31.4)$ & $979(31.5)$ & \\
\hline Referral & $4146(68.6)$ & $2016(68.6)$ & $2130(68.5)$ & \\
\hline Local resident & & & & 0.829 \\
\hline No & $2781(46.0)$ & $1347(45.8)$ & $1434(46.1)$ & \\
\hline Yes & $3266(54.0)$ & $1591(54.2)$ & $1675(53.9)$ & \\
\hline TB case & & & & 0.208 \\
\hline New & $5608(92.7)$ & $2712(92.3)$ & $2896(93.1)$ & \\
\hline Previously treated & $439(7.3)$ & $226(7.7)$ & $213(6.9)$ & \\
\hline Bacteriological confirmation & & & & $<0.001$ \\
\hline Yes & $3290(54.4)$ & $1461(49.7)$ & $1829(58.8)$ & \\
\hline DST coverage & & & & $<0.001$ \\
\hline Yes & $2573(42.6)$ & $1066(36.3)$ & $1507(48.5)$ & \\
\hline RR-TB detection & & & & 0.004 \\
\hline Yes & $156(2.6)$ & $58(2.0)$ & $98(3.2)$ & \\
\hline
\end{tabular}

PTB pulmonary tuberculosis Xpert Xpert MTB/RIF

MTB Mycobacteria of tuberculosis RIF Rifampicin

$R R$-TB Rifampicin-resistant tuberculosis DST Drug susceptibility test

*Xpert was implemented in 2018 
PTB case with DST results for RIF. The delay of RR-TB detection was defined as time (days) from the date of first visit to a TB hospital to the date RIF-resistance was detected by either conventional DST or Xpert.

Analysis was performed using $\mathrm{R}^{\circ}$ software (3.5.1). Demographic, clinical and diagnosis characteristics were analyzed and presented in frequencies and medians with an interquartile range (IQR) for categorical and continuous variables, respectively. Overall bacteriological confirmation and RR-TB detection was reported in frequencies. Comparison of frequencies was done using chi-square test, and medians using Wilcoxon rank-sum test. We estimated the probability of RR-TB detection using the Kaplan-Meier method and subgroups were compared using the log rank test. Cox proportional hazard model was used to investigate the factors associated with RR-TB detection. Unadjusted hazard ratios (HR) were calculated from a bivariate analysis. Adjusted hazard ratios were estimated by multivariate analysis. The final model was adjusted for all the variables from the bivariate analysis. A $p$ value of less than 0.05 was considered significant.

\section{Results}

Epidemiological and laboratory characteristics

There were 6357 PTB patients registered in the first half of each of 2017 and 2018. After excluding patients under 15 years old (31 cases) and those diagnosed as nontuberculous mycobacteria (NTM, 279 cases), 6047 PTB patients were included in the analysis (2017: 2938; 2018: 3109), with a median age of 44 years (IQR 27-62). The majority were male (68\%), new patients (93\%), local residents (54\%) and registered in referral hospitals $(69 \%)$, with no significant difference between 2017 and 2018.

The proportion of bacteriological confirmation and DST coverage in 2017 and 2018 were $50 \%$ vs. 59\% and $36 \%$ vs. $49 \%$, respectively. The RR-TB detection in the 2 years was 2 and 3\% among new patients; and 6 and 11\% among previously treated patients. There were $156 \mathrm{RR}$ TB patients detected, among them 119 (76\%) were new cases. Proportions of bacteriological confirmation, DST coverage and RR-TB detection were significantly higher in 2018 than in 2017 ( $p$-value < 0.05) (Table 1).

Table 2 Laboratory test results of pulmonary tuberculosis patients notified in Shanghai, China, in the first half of each of 2017 and 2018

\begin{tabular}{|c|c|c|c|c|}
\hline Characteristics & $\begin{array}{l}\text { Total } \\
\mathrm{n}(\%) \\
(n=6047) \\
\end{array}$ & $\begin{array}{l}\text { PTB patients of } 2017 \\
\mathrm{n}(\%) \\
(n=2938)\end{array}$ & $\begin{array}{l}\text { PTB patients of } 2018 \\
\mathrm{n}(\%) \\
(n=3109)\end{array}$ & $p$-value \\
\hline Sputum smear & & & & $<0.001$ \\
\hline Negative & $3784(62.6)$ & $1812(61.7)$ & $1972(63.4)$ & \\
\hline Positive & $1802(29.8)$ & $850(28.9)$ & $952(30.6)$ & \\
\hline Not available & $461(7.6)$ & $276(9.4)$ & $185(6.0)$ & \\
\hline Sputum culture & & & & $<0.001$ \\
\hline Negative & $2123(35.1)$ & $1000(34.0)$ & $1123(36.1)$ & \\
\hline Positive & $2786(46.1)$ & $1285(43.7)$ & $1501(48.3)$ & \\
\hline Not available & $1138(18.8)$ & $653(22.3)$ & $485(15.6)$ & \\
\hline Xpert result for MTB & & & & - \\
\hline Negative & $782(12.9)$ & - & $782(25.2)$ & \\
\hline Positive & $909(15.0)$ & - & $909(29.2)$ & \\
\hline Not available & $4356(72.0)$ & $2938(100)$ & $1418(45.6)$ & \\
\hline Xpert result for RIF resistance ${ }^{a}$ & & & & - \\
\hline Susceptible & $840(92.4)$ & - & $840(92.4)$ & \\
\hline Resistant & $69(7.6)$ & - & $69(7.6)$ & \\
\hline Culture-based DST result for RIF resistance ${ }^{b}$ & & & & 0.784 \\
\hline Susceptible & $2188(78.5)$ & $1008(78.4)$ & $1180(78.6)$ & \\
\hline Resistant & $118(4.2)$ & $58(4.5)$ & $60(4.0)$ & \\
\hline Not available & $480(17.2)$ & $219(17.0)$ & $261(17.4)$ & \\
\hline
\end{tabular}

PTB Pulmonary tuberculosis

Xpert Xpert MTB/RIF

MTB Mycobacteria of tuberculosis

RIF Rifampicin

DST Drug susceptibility test

${ }^{a}$ calculated among Xpert MTB positive patients

${ }^{\mathrm{b}}$ calculated among culture positive patients 


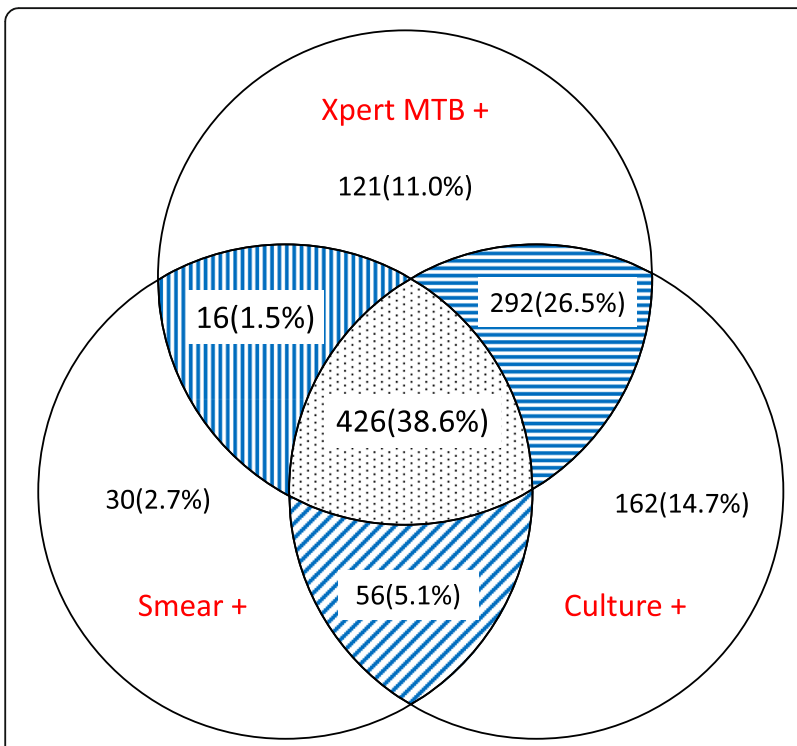

Fig. 1 Agreement of sputum smear, culture and Xpert MTB in 1103 pulmonary tuberculosis patients with all tests in Shanghai, China, in the first half of 2018. Smear + : smear positive. Culture +: culture positive. Xpert MTB +: Xpert MTB positive. The blue lines represent the agreement between two diagnostic techniques used, and the dot area shows the agreement among all three tests

\section{Effect of Xpert on bacteriological confirmation, DST} coverage and RR-TB detection in the first half of 2018 Among 1691 (54\%) PTB patients who underwent for Xpert in 2018, 909 (54\%) were MTB positive, and 69 (8\%) RIF-resistant (Table 2). A total of 1103 PTB patients completed sputum smear, culture and Xpert tests. Fig. 1 shows that Xpert failed to detect 248 (23\%) sputum smear and/or culture positive patients, but detected 121 (11\%) PTB patients who were negative by smear and culture. In addition, Xpert detected 426 out of 482 (88\%) smear-positive, culture-positive patients and 292 out of 454 (64\%) smear-negative, culture-positive patients.

Regarding DST coverage and RR-TB detection, Xpert accounted for an increase of 9 and 1\%, respectively (Fig. 2). Among 52 new patients with RIF-resistant results from Xpert, 16 (31\%) were verified with the second Xpert test: 15 remained RIF-resistant; 1 turned to RIFsensitive but culture-based DST showed RIF-resistant. Of 36 without second Xpert test, 13 were RIF-resistant, 7 RIF-sensitive according to culture-based DST and the others had no further verification.

\section{Effect of Xpert on delay of RR-TB detection}

The median of delay to RR-TB detection was 62 days (IQR 48-84) in 2017 vs. 9 days (IQR 2-45) in 2018 (Fig. 3, $p$-value $<0.001$, Wilcoxon rank-sum test). When Xpert was used, the delay to RR-TB detection was significantly shorter compared to conventional DST (Fig. 4, $p$-value $<0.001$, log-rank test).

In the multivariate model, utilization of Xpert was associated with decreased delay to RR-TB detection after adjustment for sex, age and other factors shown in the model (adjusted HR =4.62, 95\% CI 3.18-6.71) (Table 3). In addition, referral hospital and recurrent $\mathrm{TB}$ were also associated with decreased delay to RR-TB detection.

\section{Discussion}

To our knowledge, this is the first real-world, population-based study that evaluates the effect of Xpert on PTB and RIF-resistance detection at a provincial level in China. The majority of research on Xpert in China has been confined to laboratories and hospitals [23-26], focusing on the accuracy of Xpert compared to sputum smear microscopy, culture or other molecular methods. Few have studied the performance of Xpert in a routine setting. Our study indicates that within a routine setting

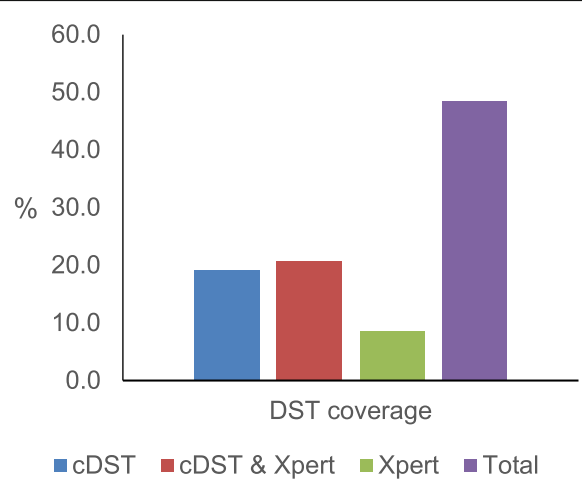

A

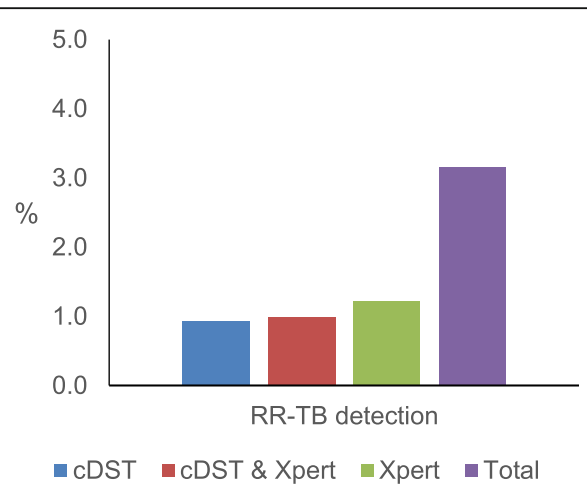

$\mathrm{B}$

Fig. 2 Drug susceptibility test coverage (A) and rifampicin-resistant tuberculosis detection (B) among pulmonary tuberculosis patients in Shanghai, China, in the first half of 2018. RR-TB = rifampicin-resistant tuberculosis. CDST = conventional drug susceptibility test. Xpert $=$ Xpert MTB/ RIF. Blue, green and red bars indicates the contribution of cDST alone, Xpert alone, and when both cDST \& Xpert were done 


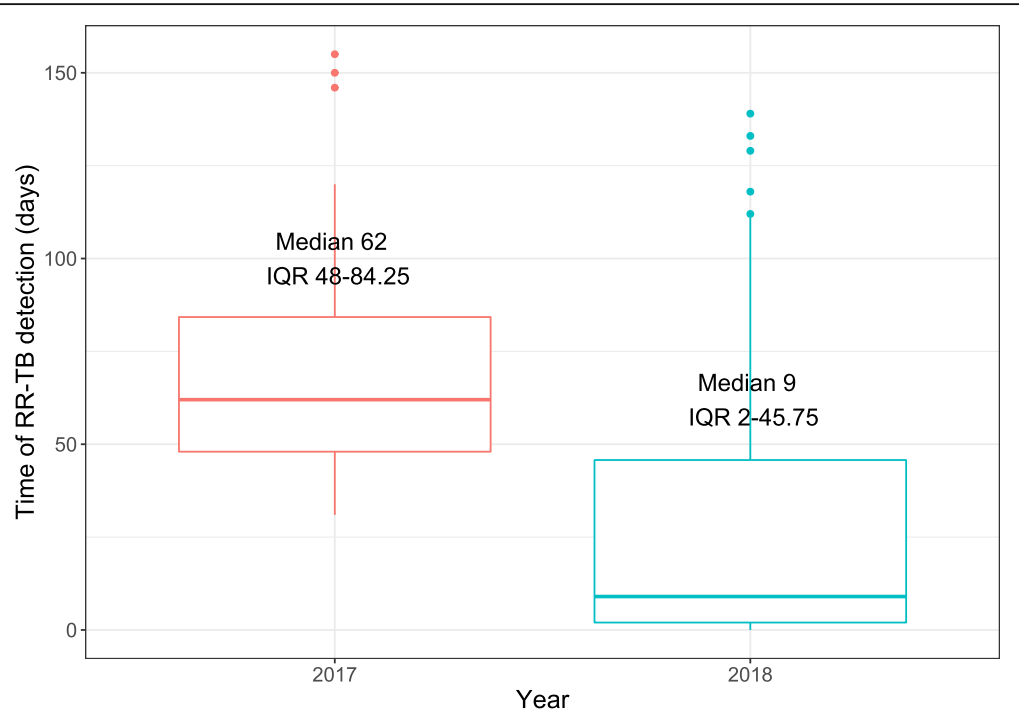

Fig. 3 Time of rifampicin-resistant pulmonary tuberculosis detection in Shanghai, China, in the first half of each of 2017 and $2018^{*}$. Time of rifampicin-resistant pulmonary tuberculosis detection: days from the first visit to tuberculosis clinics to rifampicin-resistance was reported. RR-TB $=$ rifampicin-resistant tuberculosis. ${ }^{*}$ Xpert MTB/RIF was implemented in 2018

in Shanghai, China, Xpert accounted for 11 and 9\% increase in bacteriological confirmation and DST coverage. It also increased RR-TB detection by $1 \%$ and significantly reduced delay of detection $(p$-value $<0.001)$.

Our study found a significant increase $(11 \%)$ in bacteriological confirmation when Xpert was offered to all presumptive patients. This was similar to studies in other countries and regions [12, 27-29], although the proportion varied, ranging from 5\% in Zimbabwe [29] to 59\% in Brazil [27]. We also noted the relatively low sensitivity of Xpert in this study (88\% for smear-positive, culture-positive patients; $64 \%$ for smear-negative, culture-positive patients) compared to the findings in a Cochrane review ( $98 \%$ for smearpositive, culture-positive patients; $67 \%$ for smear-negative,

\section{$-\mathrm{cDST}==$ Xpert}

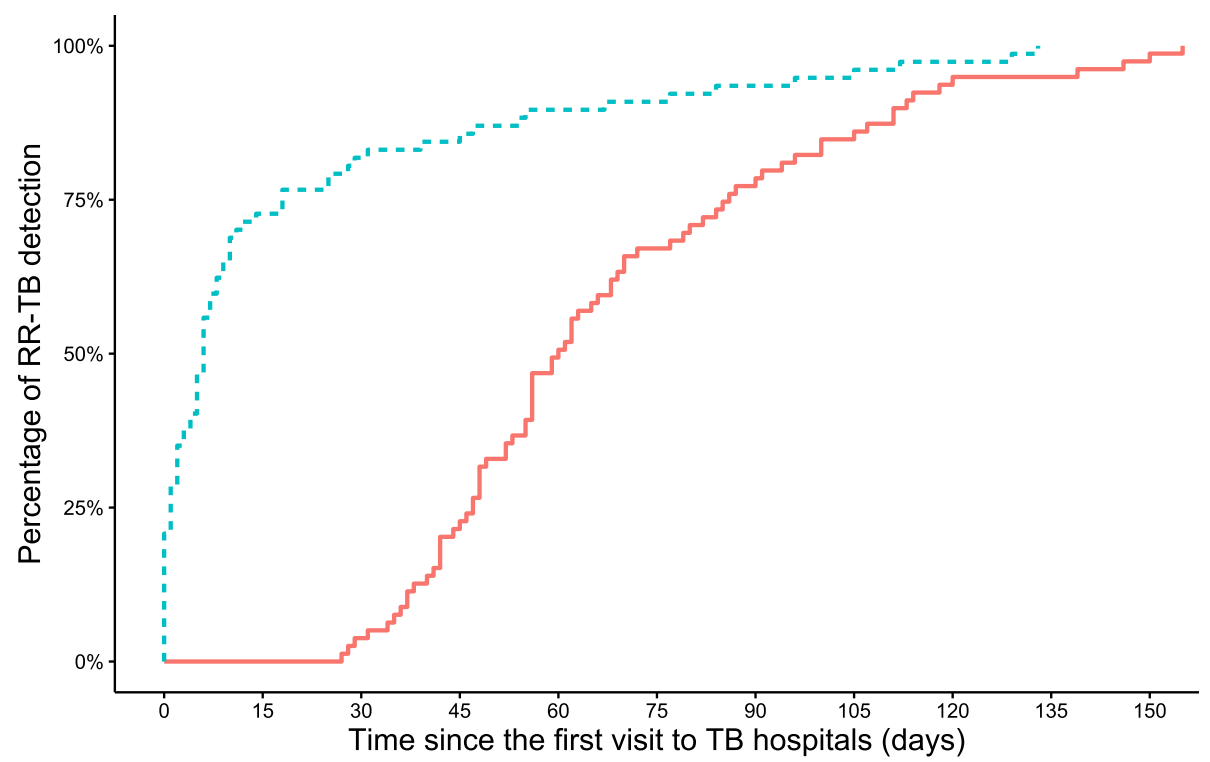

Fig. 4 Kaplan-Meier for probability of rifampicin-resistant pulmonary tuberculosis detection since the first visit to TB hospitals using Xpert $(n=77$, blue dashed line) and conventional drug susceptibility test ( $n=79$, red solid line). RR-TB $=$ rifampicin-resistant tuberculosis. CDST $=$ conventional drug susceptibility test. Xpert $=$ Xpert MTB/RIF 
Table 3 Univariate and multivariate analysis of factors associated with RR-TB detection in Shanghai, China, in the first half of each of 2017 and 2018

\begin{tabular}{|c|c|c|c|}
\hline Variables & Number of RR-TB patients $(n=156)$ & Unadjusted HR (95\%Cl) & Adjusted HR $(95 \% \mathrm{Cl})^{\mathrm{a}}$ \\
\hline \multicolumn{4}{|l|}{ Sex } \\
\hline Female & 44 & 1.00 & 1.00 \\
\hline Male & 112 & $1.05(0.73-1.51)$ & $0.88(0.59-1.31)$ \\
\hline \multicolumn{4}{|l|}{ Age } \\
\hline $15-29$ & 48 & 1.00 & 1.00 \\
\hline $30-44$ & 36 & $0.97(0.63-1.51)$ & $1.37(0.86-2.19)$ \\
\hline $45-59$ & 45 & $0.98(0.65-1.47)$ & $0.93(0.60-1.46)$ \\
\hline$>=60$ & 27 & $0.75(0.46-1.22)$ & $1.08(0.64-1.84)$ \\
\hline \multicolumn{4}{|l|}{ Hospital } \\
\hline County-level & 47 & 1.00 & 1.00 \\
\hline Referral & 109 & $2.65(1.75-4.03)$ & $2.92(1.85-4.59)$ \\
\hline \multicolumn{4}{|l|}{ Local resident } \\
\hline No & 86 & 1.00 & 1.00 \\
\hline Yes & 70 & $0.97(0.71-1.34)$ & $1.13(0.78-1.63)$ \\
\hline \multicolumn{4}{|l|}{ TB case } \\
\hline New & 119 & 1.00 & 1.00 \\
\hline Recurrent & 37 & $1.39(0.28-1.19)$ & $1.66(1.11-2.50)$ \\
\hline \multicolumn{4}{|l|}{ Sputum culture } \\
\hline Not done & 8 & 1.00 & 1.00 \\
\hline Done & 148 & $0.58(0.28-1.19)$ & $1.90(0.85-4.26)$ \\
\hline \multicolumn{4}{|l|}{ Xpert } \\
\hline Not Done & 79 & 1.00 & 1.00 \\
\hline Done & 77 & $3.26(2.35-4.53)$ & $4.62(3.18-6.71)$ \\
\hline
\end{tabular}

PTB Pulmonary tuberculosis

Xpert Xpert MTB/RIF

MTB Mycobacteria of tuberculosis

Delay of RR-TB detection: days from the first visit to a TB hospital to when rifampicin-resistance was reported

${ }^{a}$ Adjusted for the other factors shown in the multivariate model

culture-positive patients) [30]. This difference may be a result of the real-world context compared to clinical trials that control for many variables. The WHO has recommended using Xpert rather than smear microscopy and culture as the initial diagnostic test in all adults suspected of having TB if resources allow [4]. Considering that in our study Xpert missed 23\% smear microscopy or culture positive patients, further analysis is needed to identify and eliminate factors associated with the heterogeneity and improve the performance of Xpert before any modification to the diagnosis cascade should be adopted.

We also observed an increase in DST coverage and RRTB detection in our study due to the use of Xpert, which was similar to other studies [12, 31]. As the GLI and the WHO $[3,22]$ suggested, the project planned to offer new patients who had a RIF-resistant result from Xpert, a second one to reduce the rate of false positivity, but in our study only 16 (31\%) finished the second test. Nevertheless, all the 16 patients were verified as RIF-resistant. Xpert has a sensitivity of $95 \%$ and specificity of $98 \%$ compared to culture-based DST [3]. Moreover, several studies have found that part of the false positivity was attributed to low-level RIF-resistance that could not be found by culture-based DST $[3,10,11]$. Our results may indicate a high-level reliability of RIF-resistance results from Xpert in the current setting, but further evidence is needed.

In our study, the delay of RR-TB detection was dramatically reduced from 62 to 9 days after implementing Xpert. Findings in other countries [14, 32-34] have suggested that Xpert substantially decreased the delay of RR-TB treatment initiation. Our study did not include data on delay from RR-TB detection to treatment initiation. However, RR-TB detection accounted for $23 \%$ of total RR-TB treatment delay in Shanghai [16], therefore, Xpert played an important role in the improvement of RR-TB detection and transmission control. Theoretically, the laboratory procedure of Xpert takes only $2 \mathrm{~h} \mathrm{[4,}$ 13]. However, when implemented in the real world, time 
to detection might be prolonged by health service providers, logistics and patients [35]. Although there was a significant median time reduction for RR-TB detection, the fact that it still took 9 days highlights the need to identify and address the barriers in the procedure of RRTB detection to take full advantage of Xpert technology and reduce even further the time to RR-TB diagnosis.

Universal DST is one component of the End TB strategy. The WHO suggested that DST coverage reach 100 and $90 \%$ of TB patients be tested using a WHOrecommended rapid diagnostic (WRD) at the time of diagnosis by 2025 [2]. According to TB report of 2018 [1], the proportion of bacteriologically confirmed cases among PTB patients in China was $32 \%$, while the global average was $56 \%$. In addition, due to the low coverage of DST (12\% for new cases and 69\% for previously treated cases), there is a large gap between estimated and notified MDR/ RR-TB cases $(58,000$ vs. 13,069$)$. Until now, there is no national policy on the usage of a WRD as the initial diagnostic test for all people presumed to have TB.

Our findings provide critical insights about using Xpert in a routine setting in China. However, TB incidence and TB control resources vary significantly throughout the country. It is notable that the experience in Shanghai should be tailored to fit for other regions in the country accordingly.

There are some limitations in our study. Due to the observational study design, the implementation of Xpert was not controlled, $45 \%$ patients did not undergo the test. This might underestimate the effect of Xpert on the detection of PTB and RR-TB. Factors associated with low Xpert implementation should be identified to strengthen the scaling-up in the next phase. Our Xpert project was funded by local government and free for patients. Whether this is sustainable still needs to be determined. Previous studies have indicated that cost-effectiveness varied in different settings and algorithms, e.g. initial use for all presumptive TB cases or use only in sputum smear microscopy negative cases [36-39]. Finally, the HIV infection status was not available. However, Shanghai is a low HIV endemic area in China with a reported incidence of 11.8/ 100000 population in 2017, therefore, we think it would not bias our findings.

\section{Conclusion}

This study provided useful evidence regarding the potential use of Xpert in a high-burden country to increase PTB and RR-TB detection, as well as decrease RR-TB detection delay. It might be helpful for regions with similar settings when considering integrating this technique into routine PTB and RR-TB case-finding algorithms. Operational level problems should be identified and eliminated to fully utilize the benefit of rapid diagnosis by Xpert.

\section{Abbreviations}

CDC: Center for Disease Control and Prevention; Cl: Confidence intervals: DST: Drug susceptibility test; GLI: Global Laboratory Initiative; IGRA: Interferon-gamma release assay; IQR: Interquartile range; MDR/RRTB: Multidrug-resistant/rifampicin-resistant TB; MTB: Mycobacterium tuberculosis; NTM: Nontuberculous mycobacteria; PPD: Purified protein derivative; PTB: Pulmonary TB; RIF: Rifampicin; RR: Risk ratio; RR-

TB: Rifampicin-resistant TB; TB: Tuberculosis; WRD: WHO-recommended rapid diagnostic; Xpert: Xpert MTB/RIF

\section{Acknowledgements}

This research was conducted through the Structured Operational Research and Training Initiative (SORT-IT), a global partnership coordinated by the Special Programme for Research and Training in Tropical Diseases at the World Health Organization (WHO/TDR). The training model is based on a course developed jointly by the International Union Against Tuberculosis and Lung Disease (The Union) and Médecins Sans Frontières (MSF). The Specific SORT-IT programme which resulted in this publication was implemented by: Médecins Sans Frontières, Brussels Operational Centre,

Luxembourg and the Chinese Centre for Disease Control and Prevention. Mentorship and the coordination/facilitation of this SORT-IT workshop were provided through the University of Washington, Department of Global Health, USA; AMPATH, Eldoret, Kenya; Sustainable Health Systems, Sierra Leone; Universidad Pontificia Bolivariana, Colombia; Global AIDS Interfaith AIliance, USA; Centre for Operational Research, The Union, Paris, France; and the Chinese Centre for Disease Control and Prevention.

We also thank the patients and the health-care workers from all TB hospitals in Shanghai for their generous support and cooperation.

Finally, we thank to Dr. Jeffrey Edwards (Faculty of University of Washington) and Elizabeth Geoffroy (Global AIDS Interfaith Alliance) for the English review of this paper.

\section{Open access statement}

In accordance with WHO's open-access publication policy for all work funded by $\mathrm{WHO}$ or authored / co-authored by WHO staff members, the WHO retains the copyright of this publication through a Creative Commons Attribution IGO licence (http://creativecommons.org/licenses/by/3.0/igo/legalcode) which permits unrestricted use, distribution and reproduction in any medium provided the original work is properly cited.

\section{Authors' contributions}

ZW participated in the study design, data collecting, statistical analysis and wrote the first draft of the manuscript. ZVR and TL participated in the data analysis, reviewed and revised the first draft. XS, QP, ZY leaded the Xpert project and participated in the study design. ZZ, YJ, WS, JC and FY implemented the Xpert project and participated in the data collecting. All authors read and approved the final manuscript, and agreed to be accountable for all aspects of the work in ensuring that questions related to the accuracy or integrity of any part of the work are appropriately investigated and resolved.

\section{Funding}

The study was funded by Chinese National Science and Technology Major Project (2018ZX10715012 and 2017ZX10201302-006-002), National Natural Science Foundation of China (81872679) and National Key Research and Development Program of China (2017YFD0500301). The sponsors of the study had no role in study design, data collection, data analysis, data interpretation, or writing of the manuscript.

\section{Availability of data and materials}

The data sets analysed during the current study are available from the corresponding author on reasonable request.

\section{Ethics approval and consent to participate}

The study was approved by the Ethical Review Committee at Shanghai Municipal Center for Disease Control and Prevention. Since only anonymized secondary data were used, no informed consent was sought. 


\section{Competing interests}

The authors declare that they have no competing interests.

\begin{abstract}
Author details
'Department of Tuberculosis Control, Shanghai Municiple Center for Disease Control and Prevention, Shanghai, China. ${ }^{2}$ Universidad Pontificia Bolivariana, Medellín, Colombia. ${ }^{3}$ Chinese Center for Disease Control and Prevention, Beijing, China. ${ }^{4}$ Laboratory of Tuberculosis Diagnosis, Shanghai Municiple Center for Disease Control and Prevention, Shanghai, China. ${ }^{5}$ Shanghai Key Laboratory of Tuberculosis, Clinic and Research Center of Tuberculosis, Shanghai Pulmonary Hospital, Tongji University School of Medicine, Shanghai, China. ${ }^{6}$ Department of Clinical Laboratory Medicine, Shanghai Pulmonary Hospital, Tongji University School of Medicine, Shanghai, China. ${ }^{7}$ Shanghai Municiple Center for Disease Control and Prevention, Shanghai, China.
\end{abstract}

\section{Received: 23 January 2019 Accepted: 11 February 2020}

Published online: 18 February 2020

\section{References}

1. World Health Organization. Global tuberculosis report 2018. Geneva: World Health Organization; 2018

2. Uplekar M, Weil D, Lonnroth $\mathrm{K}$, Jaramillo E, Lienhardt C, Dias HM, et al. WHO's new end TB strategy. Lancet. 2015;385:1799-801. https://doi.org/10. 1016/50140-6736(15)60570-0.

3. Implementing tuberculosis diagnostics. Policy framework. Geneva: World Health Organization; 2015.

4. World Health Organization. Automated real-time nucleic acid amplification technology for rapid and simultaneous detection of tuberculosis and rifampicin resistance: Xpert MTB/RIF assay for the diagnosis of pulmonary and extrapulmonary TB in adults and children: policy update. Geneva: World Health Organization; 2013.

5. Rice JP, Seifert M, Moser KS, Rodwell TC. Performance of the Xpert MTB/RIF assay for the diagnosis of pulmonary tuberculosis and rifampin resistance in a low-incidence, high-resource setting. PLoS One. 2017;12:e0186139. https:// doi.org/10.1371/journal.pone.0186139.

6. Li S, Liu B, Peng M, Chen M, Yin W, Tang H, et al. Diagnostic accuracy of Xpert MTB/RIF for tuberculosis detection in different regions with different endemic burden: a systematic review and meta-analysis. PLoS One. 2017;12: e0180725. https://doi.org/10.1371/journal.pone.0180725.

7. Ou X, Xia H, Li Q, Pang Y, Wang S, Zhao B, et al. A feasibility study of the Xpert MTB/RIF test at the peripheral level laboratory in China. Int J Infect Dis. 2015;31:e41-6. https://doi.org/10.1016/j.jijid.2014.09.011.

8. Meyer AJ, Atuheire C, Worodria W, Kizito S, Katamba A, Sanyu I, et al. Sputum quality and diagnostic performance of GeneXpert MTB/RIF among smear-negative adults with presumed tuberculosis in Uganda. PLoS One. 2017;12:e0180572. https://doi.org/10.1371/journal.pone.0180572.

9. Shi J, Dong W, Ma Y, Liang Q, Shang Y, Wang F, et al. GeneXpert MTB/RIF outperforms mycobacterial culture in detecting mycobacterium tuberculosis from salivary sputum. Biomed Res Int. 2018;2018:1514381. https://doi.org/10. 1155/2018/1514381.

10. Van Deun A, Aung KJM, Bola V, Lebeke R, Hossain MA, De Rijk WB, et al. Rifampin drug resistance tests for tuberculosis: challenging the gold standard. J Clin Microbiol. 2013;51:2633-40. https://doi.org/10.1128/JCM. 00553-13.

11. Rigouts L, Gumusboga M, De Rijk WB, Nduwamahoro E, Uwizeye C, De Jong $B$, et al. Rifampin resistance missed in automated liquid culture system for mycobacterium tuberculosis isolates with specific rpoB mutations. I Clin Microbiol. 2013;51:2641-5. https://doi.org/10.1128/JCM.02741-12.

12. Sachdeva KS, Raizada N, Sreenivas A, Van't Hoog AH, Van Den Hof S, Dewan PK, et al. Use of Xpert MTB/RIF in decentralized public health settings and its effect on pulmonary TB and DR-TB case finding in India. PLoS One. 2015; 10:e0126065. https://doi.org/10.1371/journal.pone.0126065.

13. Boehme CC, Nicol MP, Nabeta P, Michael JS, Gotuzzo E, Tahirli R, et al. Feasibility, diagnostic accuracy, and effectiveness of decentralised use of the Xpert MTB/RIF test for diagnosis of tuberculosis and multidrug resistance: a multicentre implementation study. Lancet. 2011;377:1495-505. https://doi. org/10.1016/50140-6736(11)60438-8.

14. Kurbaniyazova G, Joncevska M, Kalon S, Kalmambetova G, Mohr T, Toktogonova A, et al. Results of Xpert ${ }^{\circledR}$ MTB/RIF implementation in
Kyrgyzstan. Int J Tuberc Lung Dis. 2017;21:333-7. https://doi.org/10.5588/ ijtld.16.0191.

15. Joshi B, Lestari T, Graham SM, Baral SC, Verma SC, Ghimire G, et al. The implementation of Xpert MTB/RIF assay for diagnosis of tuberculosis in Nepal: a mixed-methods analysis. PLoS One. 2018;13:e0201731. https://doi. org/10.1371/journal.pone.0201731.

16. Chen Y, Yuan Z, Shen X, Wu J, Wu Z, Xu B. Time to multidrug-resistant tuberculosis treatment initiation in association with treatment outcomes in Shanghai. China Antimicrob Agents Chemother. 2018;62. https://doi.org/10. 1128/AAC.02259-17.

17. Chen Y, Yuan Z, Shen X, Wu J, Wu Z, Xu B. Resistance to second-line Antituberculosis drugs and delay in drug susceptibility testing among multidrug-resistant tuberculosis patients in Shanghai. Biomed Res Int. 2016; 2016:2628913. https://doi.org/10.1155/2016/2628913.

18. Shen $X$, Xia Z, Li X, Wu J, Wang L, Li J, et al. Tuberculosis in an urban area in China: differences between urban migrants and local residents. PLoS One. 2012;7:e51133. https://doi.org/10.1371/journal.pone.0051133.

19. Yang C, Luo T, Shen X, Wu J, Gan M, Xu P, et al. Transmission of multidrugresistant mycobacterium tuberculosis in Shanghai, China: a retrospective observational study using whole-genome sequencing and epidemiological investigation. Lancet Infect Dis. 2017;17:275-84.

20. Diagnosis for pulmonary tuberculosis. People's Republic of China. 2017. http://www.nhc.gov.cn/ewebeditor/uploadfile/2017/12/20171212154852389. pdf. Accessed 16 Feb 2020.

21. Zhang Q, Wu Z, Zhang Z, Sha W, Shen X, Xiao H. Efficacy and effect of free treatment on multidrug-resistant tuberculosis. Exp Ther Med. 2016;11:77782. https://doi.org/10.3892/etm.2015.2966.

22. GLI model TB diagnostic algorithms. Global Laboratory Initiative; 2017. http://www.stoptb.org/wg/gli/assets/documents/GLI_algorithms.pdf. Accessed 16 Feb 2020

23. Huang H, Zhang Y, Li S, Wang J, Chen J, Pan Z, et al. Rifampicin resistance and multidrug-resistant tuberculosis detection using Xpert MTB/RIF in Wuhan, China: a retrospective study. Microb Drug Resist. 2018;24:675-9. https://doi.org/10.1089/mdr.2017.0114.

24. Chen C, Yang C-G, Gao X, Lu Z-Z, Tang F-X, Cheng J, et al. Communitybased active case finding for tuberculosis in rural western China: a crosssectional study. Int J Tuberc Lung Dis. 2017;21:1134-9. https://doi.org/10. 5588/ijtld.17.0123.

25. Tang T, Liu F, Lu X, Huang Q. Evaluation of GeneXpert MTB/RIF for detecting mycobacterium tuberculosis in a hospital in China. J Int Med Res. 2017:45:816-22. https://doi.org/10.1177/0300060517698618.

26. Hu PL, Bai LQ, Liu FP, Ou XC, Zhang ZY, Yi SL, et al. Evaluation of the Xpert MTB/RIF assay for diagnosis of tuberculosis and rifampin resistance in county-level laboratories in Hunan province, China. Chin Med J (Engl). 2014;127:3744-50. https://doi.org/10.3760/cma.j.issn.03666999.20141004.

27. Durovni B, Saraceni V, van den Hof S, Trajman A, Cordeiro-Santos M, Cavalcante $S$, et al. Impact of replacing smear microscopy with Xpert MTB/ RIF for diagnosing tuberculosis in Brazil: a stepped-wedge clusterrandomized trial. PLoS Med. 2014;11:e1001766. https://doi.org/10.1371/ journal.pmed. 1001766 .

28. Creswell J, Rai B, Wali R, Sudrungrot S, Adhikari LM, Pant R, et al. Introducing new tuberculosis diagnostics: the impact of Xpert ${ }^{\oplus} \mathrm{MTB} / \mathrm{RIF}$ testing on case notifications in Nepal. Int J Tuberc Lung Dis. 2015;19:545-51. https://doi.org/ 10.5588/ijtld.14.0775.

29. Jokwiro A, Timire C, Harries AD, Gwinji PT, Mulema A, Takarinda KC, et al. Has the utilisation of Xpert ${ }^{\oplus}$ MTB/RIF in Manicaland Province, Zimbabwe, improved with new guidance on whom to test? Public Heal Action. 2018;8: 124-9. https://doi.org/10.5588/pha.18.0028.

30. Steingart KR, Schiller I, Horne DJ, Pai M, Boehme CC, Dendukuri N. Xpert MTB/RIF assay for pulmonary tuberculosis and rifampicin resistance in adults. Cochrane Database Syst Rev. 2014;2014:CD009593. https://doi.org/10. 1002/14651858.CD009593.pub3.

31. Vassall A, van Kampen S, Sohn H, Michael JS, John KR, den Boon S, et al. Rapid diagnosis of tuberculosis with the Xpert MTB/RIF assay in high burden countries: a cost-effectiveness analysis. PLoS Med. 2011;8:e1001120. https://doi.org/10.1371/journal.pmed.1001120.

32. Cox HS, Daniels JF, Muller O, Nicol MP, Cox V, van Cutsem G, et al. Impact of decentralized care and the Xpert MTB/RIF test on rifampicin-resistant tuberculosis treatment initiation in Khayelitsha, South Africa. Open Forum Infect Dis. 2015;2:ofv014. https://doi.org/10.1093/ofid/ofv014. 
33. van Kampen SC, Susanto NH, Simon S, Astiti SD, Chandra R, Burhan E, et al. Effects of introducing Xpert MTB/RIF on diagnosis and treatment of drugresistant tuberculosis patients in Indonesia: a pre-post intervention study. PLoS One. 2015;10:e0123536. https://doi.org/10.1371/journal.pone.0123536.

34. Cox H, Dickson-Hall L, Ndjeka N, van't Hoog A, Grant A, Cobelens F, et al. Delays and loss to follow-up before treatment of drug-resistant tuberculosis following implementation of Xpert MTB/RIF in South Africa: a retrospective cohort study. PLoS Med. 2017;14:e1002238. https://doi.org/10.1371/journal. pmed.1002238.

35. Cohen GM, Drain PK, Noubary F, Cloete C, Bassett IV. Diagnostic delays and clinical decision making with centralized Xpert MTB/RIF testing in Durban, South Africa. J Acquir Immune Defic Syndr. 2014;67:e88-93. https://doi.org/ 10.1097/QAl.0000000000000309.

36. You JHS, Lui G, Kam KM, Lee NLS. Cost-effectiveness analysis of the Xpert MTB/RIF assay for rapid diagnosis of suspected tuberculosis in an intermediate burden area. J Inf Secur. 2015;70:409-14. https://doi.org/10. 1016/j.jinf.2014.12.015.

37. Choi HW, Miele K, Dowdy D, Shah M. Cost-effectiveness of Xpert ${ }^{\oplus M T B / R I F}$ for diagnosing pulmonary tuberculosis in the United States. Int J Tuberc Lung Dis. 2013;17:1328-35. https://doi.org/10.5588/ijtld.13.0095.

38. Pinto M, Steffen RE, Cobelens F, van den Hof S, Entringer A, Trajman A. Cost-effectiveness of the Xpert ${ }^{\oplus}$ MTB/RIF assay for tuberculosis diagnosis in Brazil. Int J Tuberc Lung Dis. 2016;20:611-8. https://doi.org/10.5588/ijtld.15. 0455.

39. Vassall A, Siapka M, Foster N, Cunnama L, Ramma L, Fielding K, et al. Costeffectiveness of Xpert MTB/RIF for tuberculosis diagnosis in South Africa: a real-world cost analysis and economic evaluation. Lancet Glob Heal. 2017;5: e710-9. https://doi.org/10.1016/S2214-109X(17)30205-X.

\section{Publisher's Note}

Springer Nature remains neutral with regard to jurisdictional claims in published maps and institutional affiliations.

Ready to submit your research? Choose BMC and benefit from:

- fast, convenient online submission

- thorough peer review by experienced researchers in your field

- rapid publication on acceptance

- support for research data, including large and complex data types

- gold Open Access which fosters wider collaboration and increased citations

- maximum visibility for your research: over $100 \mathrm{M}$ website views per year

At $\mathrm{BMC}$, research is always in progress.

Learn more biomedcentral.com/submissions 